\section{MS2-P6 Freezing without cryoprotectant}

Yvonne Thielmann ${ }^{1}$, David Quirnheim Pais ${ }^{1}$, Barbara Rathmann ${ }^{1}$, Paul Wurzinger ${ }^{2}$, Cveta Tomova ${ }^{2}$

1. Max Planck Institute of Biophysics, Molecular Membrane Biology, Max-von-Laue-Straße 3, 60438 Frankfurt, Germany

2. Leica Mikrosysteme GmbH, Hernalser Hauptstraße 219, 1170

Vienna, Austria

email: yvonne.thielmann@biophys.mpg.de

Once a protein has been crystallized successfully the crystal needs to be cryopreserved for data collection. This task can be very time consuming and may be another obstacle in the already very complex crystallization process, especially for membrane proteins. Alternatively high pressure freezing could be used to simplify the workflow with one general concept.

In cooperation with Leica Microsysteme $\mathrm{GmbH}$ a setup was created to successfully high pressure freeze protein crystals in capillaries. The setup consists of a cylinder as support that configures the nitrogen flow during freezing, and a steel base with a copper pin to hold the crystal in a polyimide capillary. Crystals can be soaked into the capillary from 96 or 24 well plates. The assembly of cylinder and base with capillary is then injected into the high pressure chamber and dropped into a dewar with liquid nitrogen. After freezing the steel base with frozen crystal and mother liquor is separated from the cylinder and kept frozen. The steel base with crystal can now be stored as usually with a plastic cap or manually mounted at a standard goniometer for analysis with an X-ray source.

Crystals of hen egg white lysozyme and Concanavalin A were successfully frozen at $200 \mathrm{MPa}$ and yielded data sets to a resolution of $1.45 \mathrm{~A}$. The structure of lysozyme was solved by molecular replacement in Phenix, followed by manual model building in Coot and refinement with Phenix. In comparison to a lysozyme structure at ambient pressure and room temperature the changes of the $\mathrm{C} \alpha$ backbone chain are negligible and comparable to another structure of a high pressure frozen lysozyme crystal (Burkhardt et al. 2013). In contrast a study of Yamada et al. (2015) showed that considerable backbone changes occur when pressures of $950 \mathrm{MPa}$ are applied on a lysozyme crystal at room temperature.

The cooperation with Leica Mikrosysteme $\mathrm{GmbH}$ is ongoing and the development for a solution of different pin lengths is ongoing. In future the frozen crystal should be automatically mountable on any synchrotron and the method should be widely applicable for usage in the crystallization community.

Burkhardt, A., Wagner, A., Warmer, M., Reimer, R., Hohenberg, H., Ren, J., Fry, E. E., Stuart, D. I. \& Meents, A. (2013). Acta Crystallogr. D Biol. Crystallogr. 69, 308-12.

Yamada, H., Nagae, T. \& Watanabe, N. (2015). Acta Crystallogr. D Biol. Crystallogr. 71, 742-53.

Keywords: high pressure freezing, crystal
MS3 Data collection and

processing software (XFELS \& synchrotrons)

Chairs: Adrian Mancuso, Thomas Schneider

MS3-P1 Computing Infrastructure, Software

Optimizatiom for High Data-Rate MX, and Real-Time Analysis

Herbert J. Bernstein ${ }^{1}$, Kaden Badalian ${ }^{2}$, Jean Jakoncic ${ }^{3}$, Edwin $\mathrm{Lazo}^{3}$, Sean McSweeney ${ }^{3}$, Wuxian $\mathrm{Shi}^{4}$, Robert M. Sweet ${ }^{3}$

1. Rochester Institute of Technology, School of Chemistry and Materials Science, Rochester NY, USA

2. SUNY Binghamton University, Binghamton, NY USA

3. Brookhaven National Laboratory, Upton, NY USA

4. Case Western University, Cleveland, OH USA

email: yayahjb@gmail.com

The very high data rates and data volumes now being seen with the new generation of pixel array x-ray detectors at synchrotron beam-lines require tuning and reorganization of computing infrastructure, and well-established processing pipelines, to make much more aggressive use of parallelism and to avoid unnecessary motion of data. Standard cluster environments, such as SGE, need to be retuned to work on much shorter time scales than usual. Standard spot-finding approaches need to be revised to make more use of "regions of interest", binning, and image summing when appropriate. Multiple conversions of images need to be avoided and compressions need to be very carefully chosen to balance the need for compact data streams against competing demands for CPU access. Data, that once could have resided on single ordinary rotating disks, now need to be in memory as much as possible, on SSDs, or when necessary, on heavily striped RAIDS of rotating disks. Networks need to be several times faster than in the past. Each of the major processing flows, such as in DIALS and XDS, need to be retuned to allow as much parallel processing of images as possible. We appreciate the cooperation shown by Dectris, the DIALS group, especially Nicholas K. Sauter, Graeme Winter, Gwyndaf Evans, and James Parkhurst, and by the XDS project, especially Kay Diederichs, as well as by collaborators at many other facilities.

Keywords: pixel-array detetectors, Eiger, cluster environments, SGE, compression, binning, summing, DIALS, XDS 